\title{
DESIGN E CONSTRUÇÃO DE NOVA INTERFACE PARA A BVS PSICOLOGIA (BVS-PŞI BRASIL) E A EXPERIÊNCIA DE CRIAÇÃO DO PROTÓTIPO DA BVS ULAPSI: FACILITANDO A VIDA DE USUÁRIOS
}

\section{Antonio Marcos Amorim}

\begin{abstract}
Resumo
Relata-se a experiência de atualização da BVS Psicologia Brasil (http://www.bvs-psi.org.br/) e criação do protótipo da BVS ULAPSI (Biblioteca Virtual da União de Entidades Latino-Americanas de Psicologia), ocorrida em 2005, pela Biblioteca do Instituto de Psicologia da USP (SBD/IPUSP) como unidade gerenciadora, em parceria com a BIREME e o Fórum das Entidades Brasileiras de Psicologia. Discutiu-se o processo de adoção de ferramentas tecnológicas e outros aspectos relacionados sempre que uma Unidade de Informação intente tal empreitada. As novas tecnologias sejam da metodologia BIREME ou softwares proprietários permitiram maior flexibilidade na inclusão de novas fontes de informação. Os profissionais técnicos e os administradores devem realizar um trabalho cooperativo na tomada de decisões, considerando a rápida introdução de novas interfaces. Assim são evitados problemas no uso de ferramentas em uma assimilação e manutenção pela equipe responsável. Em curto prazo, os profissionais devem prever as dificuldades, pois é quando ocorre o lançamento de uma biblioteca virtual.
\end{abstract}

Palavras-chave

Biblioteca virtual; Tecnologias da informação; Bibliotecas especializadas (Psicologia); Usabilidade de interfaces; Cooperação técnica; Softwares (Avaliação).

DESIGN AND CONSTRUCTION OF A NEW INTERFACE FOR THE
VIRTUAL HEALTH LIBRARY - PSYCHOLOGY (BVS-PSI BRAZIL)
AND THE EXPERIENCE OF THE BUILDING OF VIRTUAL HEALTH
LIBRARY ULAPSI'S PROTOTYPE: MAKING THE USER'S LIFE
EASIER

\begin{abstract}
Reports the experience of Virtual Health Library Psychology's update (BVS-PSI Brazil), and the building of BVS ULAPSI's prototype (Virtual Health Library of the Union of Latin American Entities of Psychology), occurred in 2005. The institutions involved are the Library of de Institute of Psychology of São Paulo University (SBD/IP/USP) as Managing Unit in partnership with BIREME and Forum of Brazilian Entities of Psychology. It discusses the process of adoption of technological tools and other related aspects, whenever one of the information units intend to carry out such project. The new technologies, whether using BIREME's methodology or proprietors softwares, allowed more flexibility in the inclusion of new information sources. The technical and administrative professionals must carry out a cooperative activity by taking decisions considering the fast introduction of new interfaces. Thus, problems in the use of tools, assimilation and maintenance from the part of the responsible team are prevented. In short term, professionals must foresee difficulties when the launching of a virtual library takes place.
\end{abstract}

\section{Keywords}

Virtual libraries; Information technologies; Specialized libraries (Psychology); Interfaces' usability; Technical cooperation; Softwares (Evaluation). 


\section{RELATO DE EXPERIÊNCIA}

\section{INTRODUÇÃO}

A Biblioteca Virtual em Saúde - Psicologia (BVS-Psi) surgiu da preocupação do Conselho Federal de Psicologia (CFP) e do Serviço de Biblioteca e Documentação do Instituto de Psicologia da Universidade de São Paulo (SBD-IP/USP) em realizar uma maior integração entre as entidades responsáveis pelo apoio ao ensino, pesquisa e atualização profissional, na área de Psicologia. Sendo o centro coordenador deste projeto, o SBD-IP/USP é considerado um centro de referência para a América Latina na área de Psicologia, dispondo de um acervo expressivo, além da mais completa coleção de periódicos nacionais e internacionais do país, tornando-se desde 1986 um centro cooperante da Rede Latino-Americana e do Caribe em Ciências da Saúde.

Conforme expõe Sampaio et al (2001), “[...] o projeto de construção da BVS-Psi iniciou-se no ano de 2000, por iniciativa conjunta do Conselho Federal de Psicologia - CFP, do SBDIP/USP e do Centro Latino-Americano de Informação em Ciências da Saúde - BIREME". Sendo lançada oficialmente em março de 2001, a BVS-Psi nasceu da iniciativa e necessidade de reunião em um portal de fontes de informações relevantes ao profissional e estudioso da Psicologia. Aqui, cabe destacar o apoio e incentivo dados pelo Conselho Federal de Psicologia, pela diretoria do SBD-IP/USP, a diretoria da BIREME / OPAS, além do Fórum das Entidades Brasileiras de Psicologia.

Mais tarde, com o sucesso do modelo da BVS-Psi, no início de 2005, tomou corpo uma iniciativa para organizar e disseminar a informação científica em Psicologia para toda a América Latina, buscando dar apoio a conhecimentos e práticas que possam responder, de modo adequado, às necessidades e urgências da sociedade da região. Com este propósito, surgia assim o projeto de criação do protótipo da BVS ULAPSI (Biblioteca Virtual de la Unión Latinoamericana de Entidades de Psicología), estreitando as relações existentes das instituições responsáveis envolvidas com o projeto no continente. 


\section{RELATO DE EXPERIÊNCIA}

\section{O DESENVOLVIMENTO DO PROJETO: ATUALIZAÇÃO DA INTERFACE BVS- PSI BRASIL E CRIAÇÃO DE PROTÓTIPO DA BVS ULAPSI}

Decorridos três anos após o lançamento da BVS-Psi - ocorrido em meados de 2004 - foi realizado um novo diagnóstico de sua interface. Entre as considerações apresentadas, foi detectada a necessidade de aperfeiçoamento no layout ou interface final de navegação aos usuários da BVS-Psi, dado que o software utilizado para este fim estava desatualizado: o BVS-Site na versão 2.4. Além disso, já havia um planejamento no sentido de melhor integrar e aperfeiçoar as interfaces e formatos de visualização das bases de dados em operação, visando sobretudo facilitar a vida dos usuários. Tais interfaces tinham diferentes graus de desenvolvimento tecnológico e estavam executando aplicações a partir de diferentes softwares - em linguagem ASP e sobretudo em CIsis, por se tratarem de bases de dados de Centro Cooperante da Rede de Ciências da Saúde, utilizando metodologia BIREME.

Naquele momento do projeto, a BVS-Psi já dispunha de uma nova versão do BVS-Site versão 3.1 - para otimizar seu uso, ampliando as possibilidades da interface aos usuários e agregando recursos da linguagem XHTML para sua manutenção, tornando-a mais dinâmica e permitindo disponibilizá-la também nos idiomas português, inglês e espanhol, e universalizando o acesso destes conhecimentos. Para concretização deste trabalho, foram realizados reuniões e trabalhos técnicos da equipe da BVS-Psi com a da BIREME para instalação de novas ferramentas disponíveis. Por este motivo então, a atualização da interface da BVS-Psi e a construção do protótipo da BVS ULAPSI seguiram um cronograma paralelo.

Como fora exposto anteriormente, a Biblioteca Virtual de Psicologia - ou BVS-Psi - estava configurada operando com o BVS-Site versão 2.4. A tela inicial do site anterior da BVS-Psi está disponível no link: http://www.bvs-psi.org.br/paginas antigas/bvs-psi.jpg.

O BVS-Site é uma aplicação gerenciadora da interface de acesso centralizado para bibliotecas virtuais, utilizando metodologias e tecnologias que geram um espaço virtual para consulta e navegação através das fontes de informação e serviços que são alimentados, gerenciados e atualizados de forma independente pelas instituições responsáveis. Esta versão ainda trazia algumas dificuldades para atualização e inclusão de fontes de informação, bem 


\section{RELATO DE EXPERIÊNCIA}

como um melhor uso de novas tecnologias, considerando-o um produto destinado a apresentar a biblioteca virtual a todos os usuários da Internet, em três idiomas. Em sua nova versão, o BVS-Site versão 3.1 permite a utilização por todos aqueles com, no mínimo, bons conhecimentos de HTML e razoáveis conhecimentos da linguagem XML/XSL (Extendible Stylesheet Language). Para todos que não o conhecem o BVS-Site versão 3.1 fora desenvolvido para trabalharmos em linguagem XSL, isto é, uma linguagem desenvolvida em XML, que permite a elaboração de novas bibliotecas virtuais usando-se de parâmetros técnicos que as tornem flexíveis, e de rápida construção e visualização, mesmo enquanto não estão prontas ou operando offline. Conforme exposto no seu manual de utilização, o BVSSite versão 3.1 é uma ferramenta onde;

\footnotetext{
o administrador pode: criar, editar e eliminar informações; relacionar dados entre si; relacionar dados com outros recursos na Internet; anexar imagens a uma determinada informação. $\mathrm{O}$ site público é altamente configurável através de modificações feitas na configuração, no entanto, requer [razoável] conhecimento de linguagem XSL(T). (BIREME).
}

Esta versão do BVS-Site permite que o processo de construção da interface das bibliotecas virtuais aos usuários seja ainda mais flexível, permitindo:

a) inclusão de fontes de informação executadas com metodologia igual ou mesmo diferente (em alguns casos) à existente na BIREME - portanto alimentadas com MicroIsis, e com possibilidade de busca simultânea pelo usuário em campos distintos de cada uma das bases de dados selecionadas;

b) a inclusão de qualquer fonte nova de informação a qualquer tempo, o que torna a biblioteca virtual um elemento dinâmico, customizável segundo as exigências e demandas de seu público usuário.

Todavia a ferramenta, como tantas outras da plataforma WWWIsis usando o XML/XSL, poderia incluir nesta versão novos aprimoramentos. Visando a todos os usuários finais - as bibliotecas desenvolvedoras - arrolamos algumas sugestões a serem consideradas:

- permitir a inserção e uso de novos idiomas - além do português, inglês e espanhol, incrementando-a. Algumas das áreas de Ciências da Saúde têm uma especificidade tal que impõe o seu uso. Entre os idiomas sugeridos, temos: alemão, italiano e francês. Assim, o produto se tornaria mais flexível e com possível aplicação mesmo em outras áreas - como as 


\section{RELATO DE EXPERIÊNCIA}

Ciências Humanas, atendendo a clientes diversos e tornando o site ou portal passível de ser exibido para outras nações. O próprio site da Organização Mundial da Saúde - OMS (http://www.who.int/) é um ótimo exemplo, quebrando barreiras lingüísticas;- ampliação do tamanho máximo de imagens que podem ser utilizadas. Atualmente não devem ultrapassar a largura / altura de 50 pixels. Muitas vezes, há a necessidade de se colocar um ícone maior, para estar mais legível ou adequado ao site da biblioteca virtual, portanto este tamanho deveria ser ampliado;

Percebemos que muitas vezes o bibliotecário responsável por uma base dados em sua biblioteca virtual, seja por não dar a devida atenção na formação continuada voltada à área de tecnologias para bibliotecas, seja pela carência no Brasil de cursos de especialização adequados, acaba ficando à mercê das vantagens permitidas por esta plataforma, no caso, um melhor uso do software CIsis. Isto ocorre freqüentemente, tornando-o desconhecedor de como funcionam os índices de recuperação e os campos a serem indexados, causando empecilhos às eventuais melhorias para a usabilidade da interface final disponível aos usuários. O próprio manual do BVS-Site na versão 3.1 recomenda, ainda de maneira elementar, que o bibliotecário pesquise quais campos podem ou são utilizados como índices, no caso os campos-chave para a indexação e recuperação de informações. A necessidade de maiores testes de usabilidade da ferramenta é de grande importância neste contexto para o projeto. Conforme aponta Bohmerwald (2005, p. 95), “[...] o teste de usabilidade é responsável por revelar como se estabelece a interação entre o usuário e o sistema, de acordo com parâmetros, como o tempo gasto para a execução de tarefas predefinidas e o caminho percorrido no site. Este teste tem o intuito de medir, com base no usuário, a facilidade de uso do site".

É também um importante alerta feito a todos os bibliotecários usuários desta ferramenta, de modo a repensarem à qual especialização a biblioteca virtual está direcionada, além da necessidade de possuírem um conhecimento consolidado do gerenciamento de suas bases de dados, permitindo assim ao sistema maior acurácia e precisão nas buscas realizadas. 


\section{RELATO DE EXPERIÊNCIA}

No caso das bibliotecas virtuais implantadas, com a necessidade de atualização das ferramentas tecnológicas, aliada ao fato de que a BVS-Psi já deveria incorporar novas fontes de informação, o BVS-Site teve a instalação de suas duas instâncias em separado, permitindo o desenvolvimento em paralelo de uma interface específica para o protótipo da BVS ULAPSI - Biblioteca Virtual de Psicologia da União Latinoamericana de Entidades de Psicologia. Entendemos aqui como instâncias os softwares responsáveis em gerenciar itens de fontes de informação e outras aplicações genéricas que dão suporte no controle do fluxo de dados entre servidor e computadores usuários espalhados pela Internet.

A equipe trabalhou de modo mais intensivo para concretizar ambos os projetos a partir de fevereiro de 2005. Como se tratavam de duas bibliotecas virtuais com conteúdo informacional semelhante, porém de escopos e objetivos distintos, a implementação das novas aplicações fora feita simultaneamente, embora no início do projeto, em dezembro de 2004, dera-se ênfase inicial às da BVS-Psi Brasil.

A sistemática adotada foi a instalação das ferramentas necessárias em um computador servidor no Conselho Federal de Psicologia (CFP) em Brasília, referentes a BVS-Psi Brasil, e outras ferramentas em servidor da BIREME, dando suporte à alimentação das bases de dados da BVS ULAPSI. Isso economizava tempo de execução, pois seu lançamento se deu em abril de 2005. Foram gerenciados e instalados os seguintes componentes ou softwares para ambas as bibliotecas virtuais:

a) o BVS-Site em um servidor do CFP em Brasília para a BVS-Psi e instalação de outro na BIREME para o protótipo da BVS ULAPSI. Este software permite gerenciar parâmetros gerais e fontes de informação da biblioteca virtual, e no primeiro caso a equipe não poupou esforços para realizar a migração da versão 2.4 para a versão 3.1 do BVS-Site;

b) a ferramenta $\mathrm{IAH}$, desenvolvida originalmente em IsisScript, e responsável por criar uma interface de pesquisa de bases de dados Isis para a Web;

c) o LILDBI-Web - um gerenciador utilizado para alimentação, no caso, de três das fontes de informação ou bases de dados já existentes: Index Psi Teses, Index Psi Periódicos TécnicoCientíficos e o Index Psi Periódicos de Divulgação; 


\section{RELATO DE EXPERIÊNCIA}

d) a instância SciELO, especialmente instalada para o projeto PEPSic - Periódicos Eletrônicos em Psicologia, uma iniciativa a este, disponibilizando periódicos eletrônicos em Psicologia em texto completo e com acesso gratuito;

e) o Localizador de Informação em Saúde - Psicologia (LIS-Psi), instalada em Brasília no servidor do CFP para o gerenciamento de inclusão de sites relevantes da área. Esta, depois de instalada, ainda de fato não entrou em plena operação de suas potencialidades, o que se dará em médio prazo, será operada e mantida pela equipe do SBD-IP/USP de modo integrado ao LIS-Regional da BIREME. Isto é, a inclusão de registros no LIS-Psi estará assinalada - literalmente - de modo sincronizado aos dados do LIS-Regional. Isto significa, que este último receberá uma marcação para cada item ou registro do LIS-Psi que seja também seu componente;

f) A aplicação DIREVE (Diretório de Eventos) - base de dados de manutenção através da Web, contendo todos os eventos de Psicologia, ou ainda outros documentos de natureza relacionada para a BVS ULAPSI, uma vez que a BVS-Psi já possuía tal aplicação congênere instalada e em operação;

Manteve-se em operação e instalados softwares de diferentes plataformas, tais como de três bases de dados - Index Psi Livros, Index Psi Filmes e Index Psi Vídeos -, operando com linguagens SQL (Structured Query Language ou Linguagem de Questões Estruturadas) e ASP (Active Server Pages) e usando o software MySQL para permitir a visualização dos dados de modo dinâmico na Web. Estas bases de dados foram desenvolvidas inicialmente por uma empresa e têm a manutenção do analista da equipe da BVS-Psi Brasil. Optou-se ainda, pela manutenção de total autonomia na publicação de documentos de ambas as bibliotecas virtuais. Foi escolhida a hospedagem dos arquivos no servidor do Conselho Federal de Psicologia em Brasília, disponibilizados basicamente em dois formatos: em PDF (Portable Document Format), da empresa Adobe e em HTML.

Este repositório de documentos inclui itens importantes, como o "Sobre a BVS", essencial ao entendimento do projeto pelo usuário de ambas bibliotecas virtuais; manuais de indexação, os tutoriais de parte das bases de dados (casos da Index Psi Periódicos Técnico-Científicos e Index Psi Teses), o item "Subsídios para o Ensino de Psicologia", auxiliando docentes e profissionais da área no exercício do ensino superior, dentre outros. Todos estes 


\section{RELATO DE EXPERIÊNCIA}

componentes, disponíveis na Web por um único meio ou portal como a Biblioteca Virtual de Saúde em Psicologia, solidificam e estabelecem novos relacionamentos entre todos os agentes envolvidos (usuários, bibliotecários, editores, autores científicos, profissionais de psicologia), maximizando a relação entre eles, e diminuindo os custos de publicação e encurtando o ciclo envolvendo a produção científica na área.

A BVS-Psi tem como forte característica de sua interface o debate democrático e a flexibilidade quanto à inclusão de itens que auxiliem o usuário, embora seja um portal consolidado há alguns anos. Um exemplo bastante ilustrativo desta virtude é a publicação nela de um manual introdutório de ajuda na pesquisa da biblioteca virtual, feito por um usuário, ou seja, com a visão de quem realmente as utiliza. O documento "Dica do usuário" (BORGES, 2004), se encontra disponível desde aquele ano até os dias atuais na BVS-Psi, no site: http://www.bvs-psi.org.br/dicausuario.htm.

Em uma fase posterior do projeto, foi feita reunião focal entre as equipes, ou seja, os técnicos da BVS-Psi e colaboradores da BIREME envolvidos, e os respectivos diretores das instituições envolvidas. Estes analisaram os rumos de desenvolvimento das atividades até aquele momento. Após um estudo de caso, foi considerado como o usuário final poderia utilizar os produtos destas bibliotecas virtuais e a apresentação destes na interface oferecida pela biblioteca virtual criada em offline com o aplicativo BVS-Site 3.1.

É da maior importância ter a ciência da estreita relação entre a equipe de bibliotecários da BVS-Psi e da Biblioteca do IPUSP com os usuários finais desta biblioteca virtual, não desprezando a cibercultura que se constrói através e com a Internet. Como bem salienta Levacov (1997, p. 127),

[..] o próprio staff da biblioteca torna-se, por sua vez, usuário remoto de outras e necessita desenvolver novos métodos de instrução bibliográfica, reinventando sua profissão e os serviços que oferece. [...] e não são mais os usuários que se encontram distantes das bibliotecas, e sim as bibliotecas que se encontram distantes dos usuários. É importante que bibliotecários participem do desenvolvimento de metaferramentas que permitam a estes usuários (com diferentes habilidades computacionais) recuperar eficientemente as informações desejadas em um ambiente informacional cada vez mais complexo. 


\section{RELATO DE EXPERIÊNCIA}

Ou seja, ao projetar uma nova interface, a equipe necessita considerar como vai se portar diante do novo meio como mecanismo de interação e trocas com seus usuários, projetando serviços adequados a estes últimos.

Tendo isso em mente, decidiu-se pela correção da ordem e da forma de apresentação dos itens, inclusive em nível hierárquico de apresentação; a inclusão de outras fontes de informação; e a realização de extratos de algumas bases de dados existentes da BIREME, otimizando a exposição de informações ao usuário da Psicologia.

Deste modo, a partir de março de 2005, tratamos de operacionalizar a customização de fontes de informação das Ciências da Saúde para a área da Psicologia, que operavam no servidor da BIREME, a serem incluídos em ambas as bibliotecas virtuais - BVS-Psi e BVS ULAPSI. Assim, foram estabelecidos critérios de elaboração de extratos destas bases de dados contemplando apenas a Psicologia e áreas relacionadas, para apresentar aos usuários um conteúdo temático pertinente. Na prática, isto fora realizado pelo uso da aplicação BVS-Site, e interfaces específicas através de técnicas de marcação e codificação em XSL / XML, ou seja, dando instruções que permitissem ao computador servidor "ler" as bases de dados de Ciências da Saúde (como por exemplo, a base LILACS) e apresentar apenas os artigos e outros documentos de conteúdo exclusivamente relacionado à área de Psicologia, seguindo outras experiências congêneres, como bem aponta o estudo de Ohira e Prado (2002).

Estabeleceram-se diretrizes para a criação de extratos das bases de dados, para disponibilizar ao usuário final uma metabusca em todas as bases desenvolvidas em plataforma IAH: seja ela específica, como o Index Psi Periódicos Técnico-Científicos, ou genérica como a base LILACS (Literatura Latinoamericana de Informação em Ciências da Saúde) e o LIS da BIREME (Localizador de Informação em Saúde Regional América Latina), isso tendo em vista o curto espaço de tempo de que dispúnhamos para lançamento de ambas as bibliotecas virtuais (BVS-Psi - nova interface - e o protótipo da BVS ULAPSI). Selecionamos então os seguintes extratos:

$\checkmark$ o Localizador de Informação em Psicologia (LIS-Psi), compreendendo atualmente mais de 70 sites referenciados, extraídos do LIS Regional da BIREME; 


\section{RELATO DE EXPERIÊNCIA}

$\checkmark$ a LILACS-Psi ou LILACS Psicologia, constituindo-se da base LILACS, excluídos aqueles documentos da América Latina que não abordem um dos temas relacionados à Psicologia e áreas afins;

$\checkmark$ o Portal de revistas científicas em Ciências da Saúde - Psicologia, um extrato formado por mais de 300 revistas científicas internacionais da área, disponíveis ou não gratuitamente e com links para cada um dos programas cooperativos de acesso a revista, quando presentes (Portal CAPES, SciELO, HINARI).

A conclusão do projeto e o lançamento das novas interfaces para estas bibliotecas virtuais, gerenciáveis de forma independente, seja em termos de cada instância ou software, bem como de cada base de dados e incluindo novas fontes de informação selecionadas pelo centro coordenador - o SBD-IP/USP - ocorreu entre 20 e 23 de abril de 2005, durante o I Congresso Latino-americano de Psicologia. Seus produtos foram o protótipo da BVS ULAPSI e a nova interface da BVS-Psi Brasil, disponíveis em seus sites: BVS-Psi Brasil - $\underline{\text { http://www.bvs- }}$ psi.org.br/ e BVS ULAPSI - http://www.ulapsi.bvsalud.org/.

\section{CONCLUSÕES}

Esta experiência indicou importantes considerações a serem feitas. Devemos atentar para fatores externos e internos à instituição responsável por uma ou mais bibliotecas virtuais, evitando-se que o projeto inicial como um todo fique comprometido, seja pela necessidade de reformulação do site, devido a inconsistências na forma como a informação científica é disponibilizada, seja pela escolha das plataformas tecnológicas e dos respectivos softwares adotados e que serão manipulados em médio e talvez longo prazos.

De um modo geral, a experiência mostrou que ao projetarmos novas bibliotecas virtuais, e ressalva seja feita a contextos bastante específicos, devemos tomar como pontos importantes:

$\checkmark$ administradores devem estar alinhados às suas equipes técnicas durante o projeto, de modo a gerenciarem de forma adequada o tempo a ser gasto e os recursos financeiros e humanos a serem empregados para execução de cada item e da empreitada como um todo; 


\section{RELATO DE EXPERIÊNCIA}

$\checkmark$ ciência de cada colaborador ou participante dos prazos a serem cumpridos, evitando que cada tarefa possa ser atrasada, causando um efeito em cascata que prejudique todo o restante das tarefas envolvidas;

$\checkmark$ senso de empreendorismo a toda à equipe envolvida, incluindo treinamento adequado e suficiente para a geração dos produtos finais desejados, seja na seleção e inclusão de seus recursos informacionais, seja para agregar know-how técnico a toda equipe;

$\checkmark$ visão estratégica do administrador e, sempre quando possível, de seus subordinados. Isto cria um ambiente organizacional permitindo durante o processo que hajam alterações de rota, envolvendo qualquer componente do sistema, caso este tenha alguma dificuldade de ordem técnica ou logística com a implantação e transferência de novos conhecimentos;

$\checkmark$ dotar o projeto de diversos profissionais (analistas, bibliotecários e webdesigners) com experiência na elaboração de bibliotecas virtuais, alinhados às necessidades dos usuários e à visão estratégica do projeto conforme este esteja sendo desenvolvido, não prejudicando assim sua execução e finalização;

$\checkmark$ incentivo ao trabalho cooperativo entre todas as equipes, possibilitando cada vez maior sinergia, seja do ponto de vista técnico-operacional ou administrativo;

$\checkmark$ rastreamento do maior número possível de potenciais instituições patrocinadoras e de apoio logístico, evitando a carência de recursos financeiros e/ou materiais, e a sobrecarga de tarefas. Novas alternativas empregadas para obtenção de recursos no contexto da realidade dos órgãos públicos devem ser consideradas, sempre quando possível.

A implantação de nova interface para a BVS-Psi e a criação do protótipo da BVS ULAPSI embora tenham sido finalizadas, jamais podem ser vistas como um fim em si mesmo. Considerando todos os usuários potenciais dos recursos informacionais nestas disponíveis, é algo sempre inacabado. Todavia, o projeto foi uma experiência agregadora de conhecimentos para toda a equipe envolvida.

É necessário ressalvar que este paper expôs determinados aspectos - o lado técnico e administrativo - de desenvolvimento do projeto, e por isso mesmo não se esgotam outras leituras possíveis. Não cabe aqui, embora o enfoque técnico seja evidente, uma postura de mero encantamento pelas principais tecnologias empregadas na experiência. Agregar recursos informacionais e utilizar novas ferramentas tecnológicas ressalve-se, jamais estará 


\section{RELATO DE EXPERIÊNCIA}

numa relação causal direta de possibilitar o aprendizado imediato por parte dos interessados o aluno, psicólogo, pesquisador, professor, enfim, quaisquer usuários.

A experiência e o uso destas ferramentas tecnológicas além disso, devem analisar também as implicações educacionais e de conteúdo envolvidos nessas bibliotecas virtuais, e é preciso tempo de observação e a avaliação sob outro enfoque para saber se, no processo de assimilação do conhecimento, estas bibliotecas virtuais estão contribuindo qualitativamente enquanto instrumento facilitador da aprendizagem de conteúdos pelos usuários, sendo matéria de um outro artigo. Todavia, a experiência expõe fatores decisivos para a concretização de uma ferramenta enquanto etapa imprescindível para uma posterior conquista de melhores usos da tecnologia pela e para a pesquisa científica. Tudo isto antecede enfim o primeiro passo: aquele a ser dado sempre pelos profissionais da informação: a eterna busca e questionamento do saber existente para a construção de novos saberes em sua área de atuação.

\section{REFERÊNCIAS}

BIREME - CENTRO LATINO-AMERICANO E DO CARIBE DE INFORMAÇÃO EM CIÊNCIAS DA SAÚDE. Manual de administração BVS-SITE. Disponível em: $<$ http://productos.bvsalud.org/dol/docsonline/1/5/BVSsite3.1.pdf $>$. Acesso em: 15 de mar. 2005.

BOHMERWALD, P. Uma proposta metodológica para avaliação de bibliotecas digitais: usabilidade e comportamento de busca por informação na Biblioteca Digital da PUC-Minas. Ciência da Informação, Brasília, v. 34, n. 1, p. 95-103, jan./abr. 2005. Disponível em:

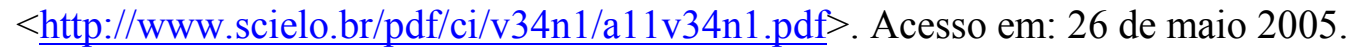

BORGES, V. C. Dica do usuário: acesso às bases de dados de periódicos científicos. São Paulo, Biblioteca Virtual de Psicologia, 2004. Disponível em: <http://www.bvspsi.org.br/dicausuario.htm>. Acesso em: 13 de maio 2005.

LEVACOV, M. Bibliotecas virtuais: (r)evolução?. Ciência da Informação, Brasília, v. 26, n. 2, p. 125-135, maio/ago. 1997. Disponível em: $<$ http://www.scielo.br/pdf/ci/v26n2/v26n22.pdf $>$. Acesso em: 12 de maio 2005.

OHIRA, M. L. B.; PRADO, N. S. Bibliotecas virtuais e digitais: análise de artigos de periódicos brasileiros (1995/2000). Ciência da Informação, Brasília, v. 31, n. 1, p. 61-74, jan./abr. 2002. Disponível em: <http://www.scielo.br/pdf/ci/v31n1/a07v31n1.pdf $>$. Acesso em: 18 de abr. 2005. 


\section{RELATO DE EXPERIÊNCIA}

SAMPAIO, M. I. C. et al. A biblioteca virtual em saúde - Psicologia (BVS - Psi). In: ENCONTRO NACIONAL DE EDITORES CIENTÍFICOS, 8., 2001, Atibaia. Resumos... Atibaia: Associação Brasileira de Editores Científicos, 2001. p. 71.

\section{Antonio Marcos Amorim}

Bibliotecário, Serviço de Biblioteca e Documentação - Instituto de Psicologia da USP

Gerente da Biblioteca Virtual de Saúde - Psicologia (BVS-Psi Brasil) e da BVS ULAPSI

E-mail: amarcos@usp.br

Telefones: (11) 3091-4392 (USP)

Currículo LATTES: http://buscatextual.cnpq.br/buscatextual/visualizacv.jsp?id=K4799722Z6

Artigo aceito para publicação em janeiro de 2006. 